\title{
Strates
}

STRATES Matériaux pour la recherche en sciences sociales

14 | 2008

Espaces du quotidien

\section{Petite chronique de la vie quotidienne à la Algaida (Andalousie occidentale)}

Yves Luginbühl et Marianne Cohen

\section{Q OpenEdition \\ 1 Journals}

Édition électronique

URL : http://journals.openedition.org/strates/6667

DOI : $10.4000 /$ strates. 6667

ISSN : $1777-5442$

Éditeur

Laboratoire Ladyss

Édition imprimée

Date de publication : 1 janvier 2008

Pagination : 69-84

ISBN : 0768-8067

ISSN : 0768-8067

Référence électronique

Yves Luginbühl et Marianne Cohen, «Petite chronique de la vie quotidienne à la Algaida (Andalousie occidentale) », Strates [En ligne], 14 | 2008, mis en ligne le 04 mars 2013, consulté le 08 septembre 2020. URL : http://journals.openedition.org/strates/6667; DOI : https://doi.org/10.4000/strates.6667

Ce document a été généré automatiquement le 8 septembre 2020

Tous droits réservés 


\title{
Petite chronique de la vie quotidienne à la Algaida (Andalousie occidentale)
}

\author{
Yves Luginbühl et Marianne Cohen
}

\section{Introduction}

1 Tout d'abord centrée sur la question de la gestion de l'eau et de la perception du risque hydraulique en milieu semi-aride ${ }^{1}$, l'analyse de ce territoire andalou de production maraîchère s'est avérée particulièrement intéressante pour tenter d'expliciter les facteurs qui interviennent dans le déroulement de la vie quotidienne de ses habitants. Les enquêtes de terrain effectuées entre septembre 2004 et avril 2005 ont permis en effet d'observer la vie des agriculteurs et de leur famille, confrontés non seulement au problème de la production horticole dans un milieu très difficile, aux sols peu propices a priori à la croissance des végétaux et surtout caractérisé par des difficultés d'accès à l'eau. Mais elles ont révélé également une véritable course d'obstacles à laquelle ces habitants sont obligés de se livrer au jour le jour pour parvenir à un niveau de vie et de confort à peine satisfaisant.

Car c'est bien ce qui frappe ici, lorsque l'on est amené à observer les déplacements dans cette colonie agraire, les multiples allées et venues des voitures, des motos ou des vélos, des camions frigorifiques, des tracteurs ou des engins agricoles ou des autobus, les jets des asperseurs. Lorsque l'on entend le murmure incessant des bruits de l'activité grouillante des maraîchers et des négociants. Lorsque l'on sent les odeurs fétides des amendements des sols, des produits phytosanitaires ou des feux de déchets divers. Lorsque l'on voit les reflets de la lumière crue du sud sur les revêtements plastiques des serres et que l'on ressent la morsure du soleil sur la peau ou du sable soulevé par le vent venant de l'Atlantique tout proche. C'est en effet la traduction sensorielle de la vie quotidienne d'un millier de familles, arc-boutées contre la multitude de contraintes d'une vie dure et que l'on pourrait penser, d'emblée, insurmontable. Et pourtant, ils 
vivent, ils cultivent, ils font la fête, ils ont des enfants, ils pensent à un avenir possible pour eux, mais à quel prix !

Quel est ce milieu où ils vivent? Qui sont-ils ? Comment font-ils pour vivre et espérer? Il faut donc comprendre ce milieu, savoir qui sont ces familles qui vivent là, et comment elles se démêlent avec ces multiples contraintes pour commencer à envisager une vie possible dans ce paysage qui semble totalement artificiel et reconstruit par presque un siècle de travail incessant du sol.

\section{Un milieu continuellement recomposé par le travail quotidien}

4 La colonie agraire de Monte Algaida se situe à l'embouchure du Guadalquivir, à quelques kilomètres au nord-est de la ville de San Lucar de Barrameda ${ }^{2}$, en limite des marais immenses du fleuve (figure 1), sur une bande de terrain sableux correspondant à des dunes stabilisées (Ambrosy, 1981, Bejarano-Palma 1997). En limite nord de la colonie, la route se transforme en une méchante piste, induisant un certain isolement géographique. À l'origine, ces terres étaient exploitées collectivement selon le système de la dehesa ${ }^{3}$ (depuis le $\mathrm{XV}^{\mathrm{e}}$ siècle). Elles sont décrites par Velasquez-Gaztelu en 1760 comme des terrains sableux, bien ombragés, où abonde l'eau douce (l'auteur signale un puits en 1526). À partir de 1803, des plantations de pins pignons y furent réalisées. Lors de la création de la colonie, ces terrains furent partiellement défrichés et livrés aux nouveaux agriculteurs, après un aménagement sommaire : une voie centrale Nord Sud fut tracée, ainsi qu'une dizaine de voies transversales perpendiculaires qui permettaient d'organiser le territoire en lots de un ou deux hectares par famille (Torrejon y Boneta, 1914). Ce lot familial comportait en outre un corps d'habitation et un petit bâtiment d'exploitation; cette architecture subsiste encore, mais de nombreuses maisons, qui étaient très rudimentaires, ont été modifiées peu à peu (figure 2). Mais les agriculteurs n'ont pas modifié seulement les maisons mais aussi profondément transformé leur milieu par leur travail quotidien. Ils ont commencé par la réalisation des navazos ${ }^{4}$, sorte de parcelles encaissées par creusement d'une couche de terre suffisante pour que les racines des plantes cultivées soient rapprochées de la nappe phréatique. La terre sableuse décaissée servit à confectionner des talus plantés de yuccas et de figuiers de Barbarie, en bordure des parcelles, qui se trouvaient ainsi protégées des vents desséchants qui soufflent soit du ponente (ouest), soit du levante (est à sud-est) et qui peuvent causer la mort de certaines plantes cultivées fragiles au stade jeune comme les carottes. Le micro-climat des navazos permettait ainsi une production intensive et précoce (Ambrosy, 1981 ; Roldan-Cruz, 1994). 
Figure 1. Bassin versant et bassins de gestion de l'eau.

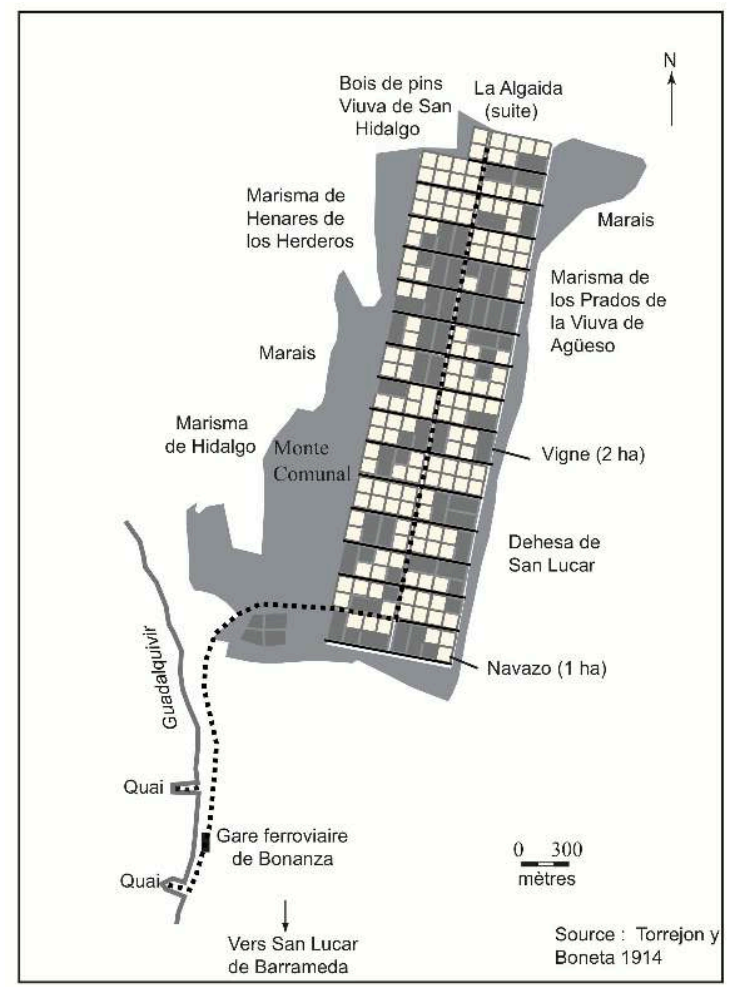

5 Les premières années de maraîchage pratiqué à la pioche ou la bêche furent extrêmement pénibles, car il fallait chaque année retourner le sol qui était rapidement infesté de nématodes et l'usage des pesticides était encore inexistant. Les sols ont donc été profondément remaniés ${ }^{5}$. Les productions étaient alors conduites au port de Bonanza par un petit train à voie étroite (figure 2) qui permettait de rapporter de la ville les déjections des fosses septiques urbaines pour amender les sols. C'était ces mêmes agriculteurs qui se chargeaient de ces corvées de curage de fosses septiques. Peu à peu, dans les parcelles des navazos, furent creusés des puits qui permirent d'arroser les plants des légumes, à l'aide de jarres, notamment pendant les mois d'été pendant lesquels le niveau de la nappe baissait. Ces travaux, réalisés annuellement, saisonnièrement ou quotidiennement, restent en mémoire aux plus anciens comme des tâches dignes du bagne. 
Figure 2. La colonie de Monte Algaida lors de sa création.

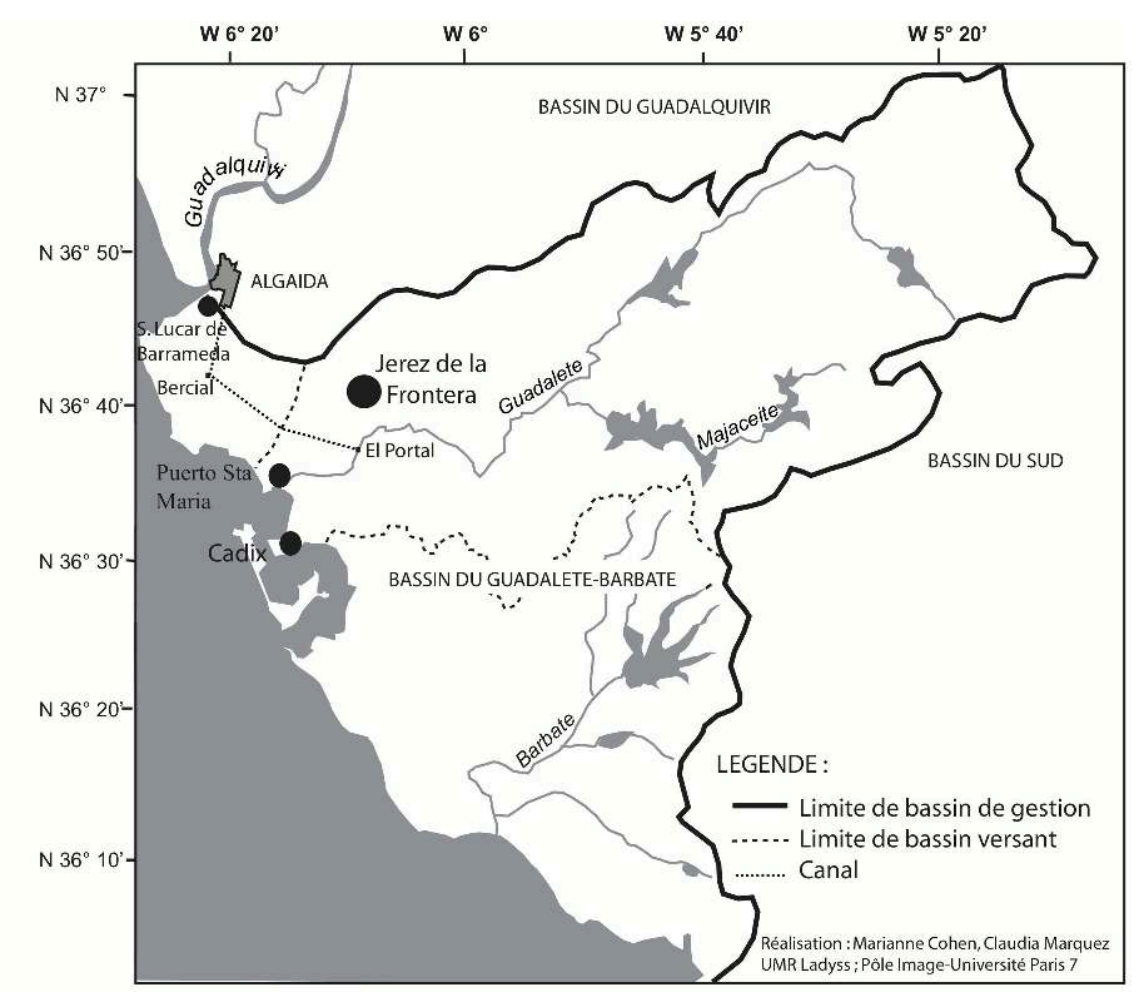

6 Puis, dans les années 1970, la mécanisation arriva et les agriculteurs utilisèrent des pompes thermiques à moteur diesel pour mettre l'eau sous pression et arroser par aspersion. La consommation d'eau augmenta et la conséquence fut assez rapide : en quelques années, au début des années 1980, la nappe salée de l'embouchure du Guadalquivir entra dans la nappe d'eau douce et les agriculteurs se trouvèrent confrontés à une nouvelle contrainte, l'eau d'irrigation saumâtre (Ministerio de Medio Ambiente, 1994). Il fallut alors trouver une solution qui leur permette de poursuivre leur activité : ils demandèrent le raccordement de leur réseau au canal du Guadalete qui arrive à une trentaine de kilomètres, jusqu'à la lagune du Bercial (figure 1), bien que leur territoire soit situé dans le bassin-versant du Guadalquivir, mais trop en marge pour imaginer un prolongement des canaux qui apportent l'eau aux zones de marais.

Une nouvelle lutte commença, avec les institutions de la très puissante Confederación Hidrográfica del Guadalquivir, qui avait autorité sur tous les bassins versants atlantiques d'Andalousie. En même temps, fut entrepris l'aménagement des marais pour agrandir la taille de la colonie et rentabiliser les travaux d'adduction. Les négociations durèrent au moins sept ans, et finalement, une canalisation fut installée jusqu'à la limite de la colonie, du côté des marais, mais sans pression (eau gravitaire). Son débit est très faible et l'eau n'arrive pas tous les jours, la canalisation étant soumise à de nombreuses pannes. En outre, la prise d'eau du canal du Guadalete se fait sur un barrage situé sur le fleuve Guadalete à proximité de la mer (El Portal, figure 1) et il arrive qu'à marée haute, l'eau de mer submerge le mur du barrage, entraînant un taux de salinité dans l'eau d'irrigation qui dépasse le seuil négatif absolu de $3 \mathrm{~ms} / \mathrm{cm}$. Dans ce cas la fourniture d'eau est interrompue pour plusieurs jours (figure 3). 
8 La vie quotidienne d'Algaida trouva ainsi une nouvelle forme de contraintes apportée par la mécanisation et les effets pervers de la technique :

- d'une part, à la lourdeur des tâches manuelles, " à la force des bras ", voire discriminantes comme le curage des fosses septiques qui fait remonter des souvenirs de travail ingrats du passé s'est substituée la mécanisation et l'usage de produits chimiques ; apparemment, ces pratiques entraînent un allégement du travail manuel, mais il est largement compensé par l'agrandissement des terres, la multiplication des cycles de culture (notamment avec le développement des serres, suite au « boum » de l'œillet, figure 4) et donc l'augmentation des tâches journalières.

- l'allégement des tâches quotidiennes grâce au progrès technique a eu des conséquences imprévues : des effets pervers et d'accumulation, comme la salinisation des nappes qui a été accélérée par l'augmentation de la dépense en eau due à sa mécanisation, ou l'exposition aux vents, accrue sur les marais et dans la colonie suite à la destruction des navazos, et qui n'est que partiellement compensée par la multiplication des serres ${ }^{6}$. Par ailleurs, la nécessité de produire davantage pour compenser les bas prix de vente des légumes à contre saison et en deux récoltes par an (au moins) et la culture des œillets ont multiplié les risques d'infestation des sols par les nématodes.

Figure 3. pH et conductivité électrique de l'eau du canal d'irrigation à Algaida.

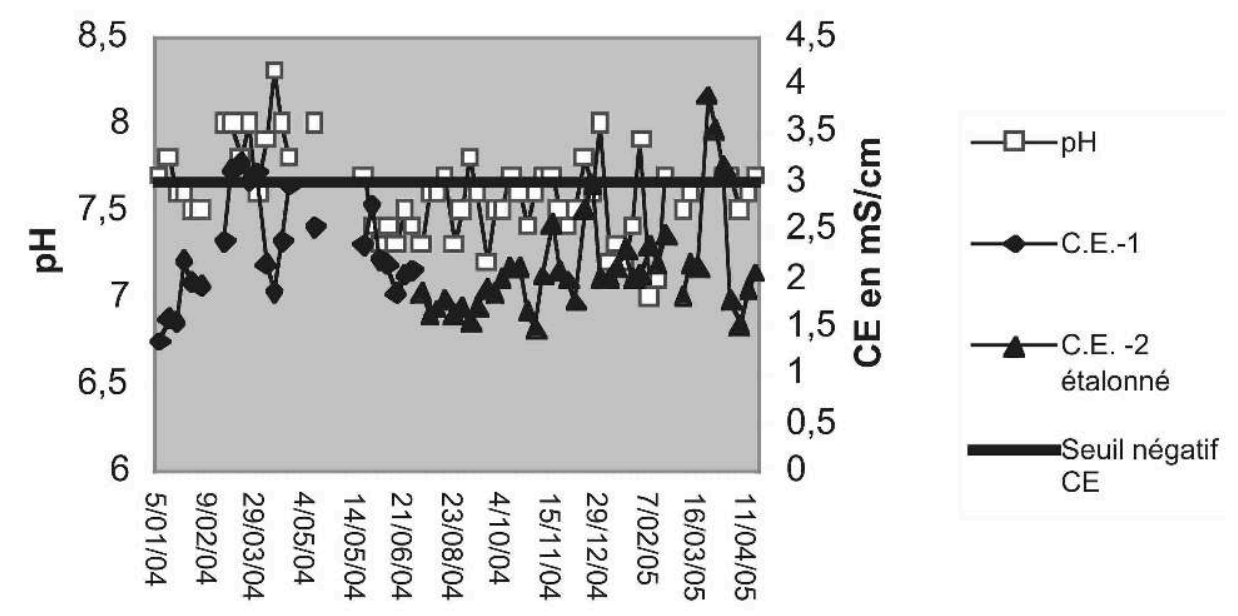

Source : Communauté d'irriguants,Algaida.

9 Même si l'on peut admettre que désormais, une eau de qualité moyenne arrive à la colonie de Monte Algaida, il faut compter au quotidien avec les problèmes phytosanitaires. Souvent élevées dans des serres ou dans des espaces assez confinés, les plantes potagères ou florales cultivées sont soumises à des infestations de parasites impromptues, fréquentes et tenaces. Un pesticide n'a pas ici la vie longue : très vite, les parasites végétaux ou animaux (champignons ou insectes) deviennent résistants aux produits du commerce et il faut attendre que la recherche agronomique ait trouvé une nouvelle molécule qui sera efficace sur les parasites qui ont muté7. De plus, les nouvelles normes européennes exigent que des limites de concentration soient respectées, ce qui réduit selon les agriculteurs l'efficacité des pesticides. S'installe ainsi une véritable course contre la montre entre les agriculteurs, les vendeurs de produits phytosanitaires, l'établissement des normes et les infestations de parasites. 


\section{Une société locale atomisée et placée dès l'origine sous la contrainte} de San Lucar, dans le cadre de la Loi de Colonisation (encadré 1). Ces terres, propriété de la grande famille de Medina Sidonia ${ }^{8}$ suite à la Reconquête, avaient été cédées en 1445 aux habitants de San Lucar, qui y conduisaient leurs troupeaux. En 1910, 462 ha de terres collectives furent déclassifiés pour y installer 194 familles, réparties comme suit : 138 lots familiaux d'1 ha consacrés aux cultures légumières (navazos), 56 lots familiaux de 2 ha destinés à la vigne, 208 ha de terrains communaux (bois, voirie et places publiques, figure 2, Torrejon y Boneta 1914, Ambrosy, 1981).

Encadré 1 : Monte Algaida et la loi de Colonisation de 1907

Monte Algaida fait partie des nombreux périmètres irrigués qui furent installés sur le territoire espagnol et surtout en Andalousie à partir du début du $\mathrm{XX}^{\mathrm{e}}$ siècle par l'Instituto Nacional de Colonización créé par l'État pour remettre en cultures de nombreuses terres incultes. C'était après la défaite de Cuba où l'Espagne perdit sa dernière colonie ; cet événement fut très mal vécu, comme une honte, par l'élite politique espagnole qui y vit l'incapacité d'un ancien État colonial puissant de conserver ses territoires outre-mer à un moment où les autres nations européennes confortaient au contraire leur empire colonial. La solution qui fut alors préconisée consista en la re-colonisation du territoire intérieur du pays, qui avait été abandonné par ses élites plus soucieuses de faire fructifier les investissements réalisés de l'autre côté de l'Atlantique. En Andalousie, où de vastes espaces de marais subsistaient incultes, de grands travaux furent entrepris pour drainer les sols et les dessaler afin d'y cultiver du coton, du riz ou d'autres spéculations agricoles destinées à l'agro-industrie.

Davantage qu'à distribuer ou coloniser la terre, ces établissements visaient à fixer des familles plutôt pauvres d'ouvriers agricoles ${ }^{9}$ qui devaient conforter la réserve de maind'œuvre des grandes propriétés latifondiaires de la région et principalement des grandes bodegas du vin de Jerez, d'où, peut-être, le projet de faire cultiver la vigne par les colons.

Les familles reçurent un sac de semences et un âne, outre la terre et la maison. Selon sa taille, le lot était dévolu à la culture des légumes ou de la vigne, ce qui fut l'une des premières contraintes; mais les agriculteurs abandonnèrent rapidement la culture viticole, inadaptée aux sols sableux pauvres, pour celle des légumes. Le nombre de familles augmentant, les espaces communaux boisés, disponibles aux marges des terrains agricoles de la colonie, qui étaient réservés au début à l'élevage des animaux de trait, ont été défrichés et allotis. Avec la mécanisation et la spécialisation dans le maraîchage, ils ont été reconvertis en terrains agricoles (figures 2 et 4). Parallèlement, à partir des années 1980, c'est vers les terres des marais que l'extension fut possible grâce aux grands travaux d'aménagement hydrauliques engagés par l'État (600 ha : figure 4). 
Figure 4. La colonie de Monte Algaida aujourd'hui.

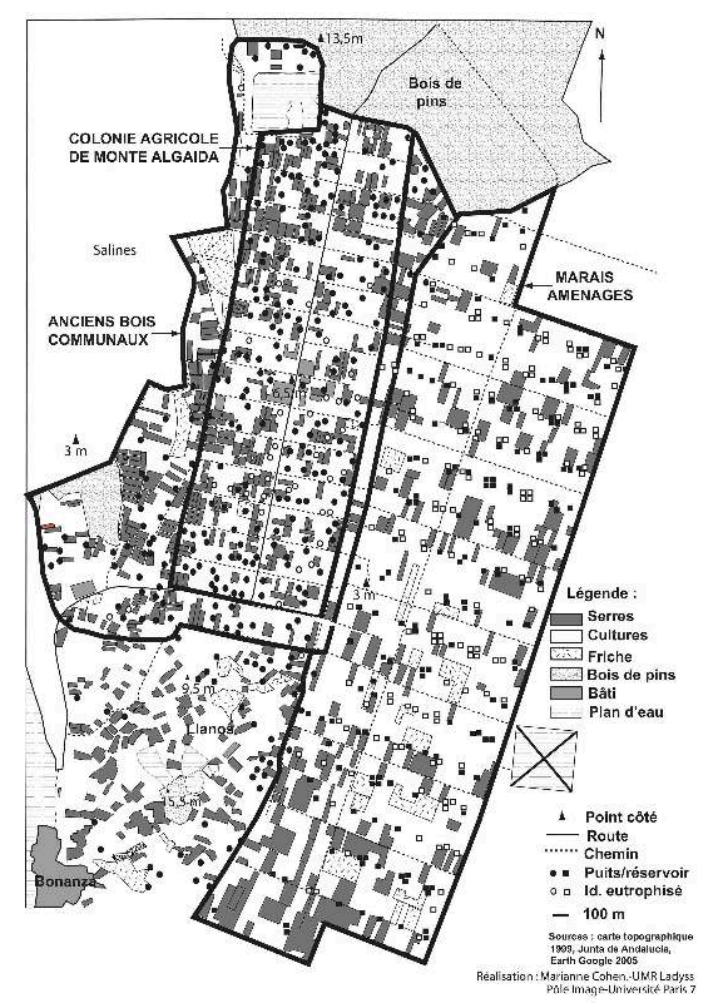

13 Il aurait été nécessaire de réaliser une recherche sur les modes de répartition de ces nouveaux terrains, car il est vraisemblable que tous ne purent en bénéficier. Dès cette époque, des stratégies familiales d'agrandissement des parcelles se mirent en place, révélant une société locale conflictuelle, soumise à un jeu de négociations souterraines entre certains individus qui surent profiter de leur position dans la société locale.

Les agrandissements sur les marais témoignent également de ces inégalités de partage, qui se manifestèrent par des acquisitions disparates. Mais ce qui conduisit à cette situation fut sans doute le mode technique de reconstitution des sols dans les marais. En effet, les terres des marais sont limoneuses (figure 5, 70-80 cm de profondeur), peu perméables, voire salines (CE à $70-80 \mathrm{~cm}$ de profondeur : 1,7 à 3,7 ms/cm), autres contraintes. Telles quelles, elles n'étaient pas aptes à la culture des plantes potagères. Il fallut apporter une couche de terre sableuse de manière à obtenir un sol perméable et suffisamment souple pour le maraîchage. Une excavation fut pratiquée au nord de la colonie, une autre au sud, pour fournir le sable ${ }^{10}$, les derniers talus furent arasés. 
Figure 5. Granulométrie des sols de marais aménagés chez cinq agriculteurs d'Algaida.

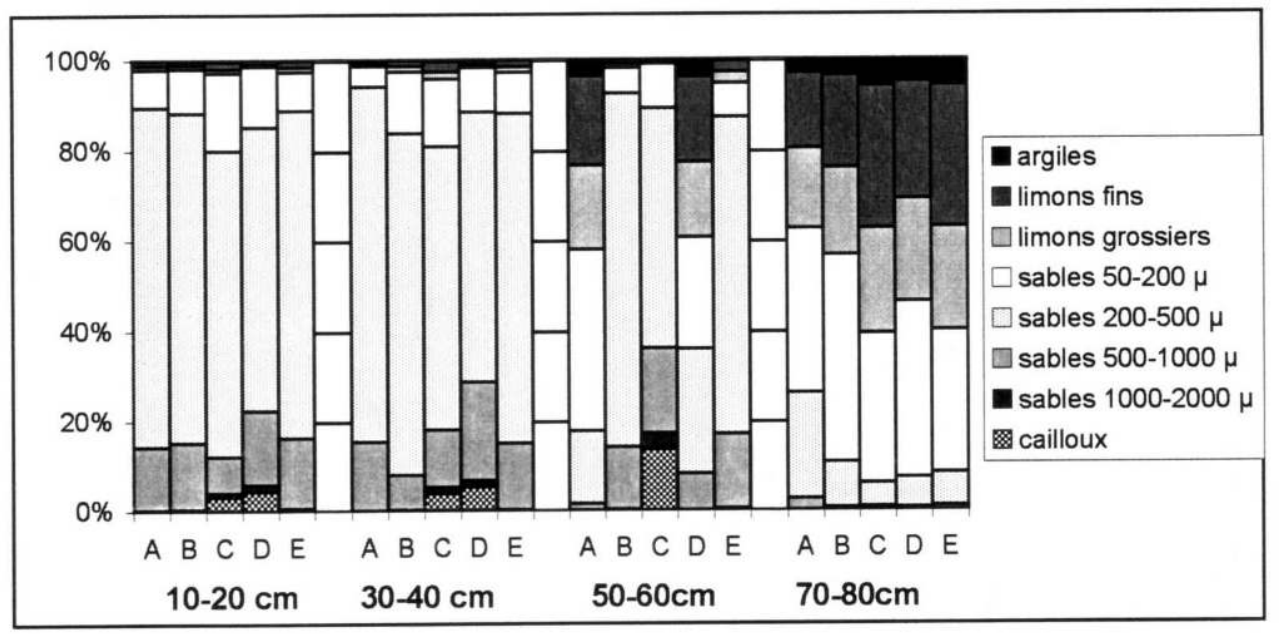

Les agriculteurs durent payer ce sable et c'est ainsi que l'inégalité prit corps, les uns réussissant à obtenir une couche de $80 \mathrm{~cm}$ de nouveau sol, alors que d'autres devaient se contenter de $40 \mathrm{~cm}$. La texture de la couche de sable est en outre plus ou moins grossière. La figure 5 donne une idée, sans prétention statistique, de la variabilité des situations. Cette différence de traitement est bien évidemment à mettre au compte des jeux de négociation des agriculteurs avec les entreprises qui extrayaient le sable. En tout cas, elle est mal vécue par ceux dont le sol n'atteint pas l'épaisseur souhaitée, d'autant que les infrastructures de drainage ne peuvent pallier à ces insuffisances. Les difficultés de conduite de la culture sur ces terrains de qualité médiocre sont rencontrées quotidiennement par les agriculteurs concernés. D'autres, plus malchanceux encore, n'ont pas obtenu de parcelle aménagée sur les marais, où l'on observe des friches (figure 4) et doivent donc se contenter du lot dont ils ont hérité.

La colonie ayant un siècle d'existence, elle a été soumise au partage des parcelles par les héritages, parallèlement aux agrandissements dont ont bénéficié certains. Aujourd'hui, 941 familles se partagent 1054 ha. Mais ces familles possèdent un fonds de terre très inégal qui peut aller de quelques centaines de $m^{2}$ à plusieurs hectares (figure 6). De plus, le statut foncier de ces terres est souvent incertain (indivision, régularisation en cours), de même que le statut des agriculteurs (tous ne sont pas "déclarés »). C'est bien évidemment une situation qui ne permet pas de renforcer la cohésion sociale, les uns se considérant frustrés par rapport aux autres dont la propriété atteint 3 à 4 hectares; mais en fait ces derniers sont rares. Ils ont souvent été chargés de responsabilités dans la communauté des agriculteurs, et notamment dans la Comunidad de regantes qui joue un rôle important pour le partage de l'eau, la fixation du prix du $\mathrm{m}^{3}$ utilisé pour l'irrigation, etc. Ce sont évidemment des postes convoités qui permettent de conduire des stratégies permettant de bénéficier d'un avantage. Autant de motifs de frustration pour les autres, ce qui ne va pas dans le sens d'une entente parfaite, mais bien plutôt vers le renforcement des mécontentements. 
Figure 6. Distribution des tailles d'exploitations à Algaida.

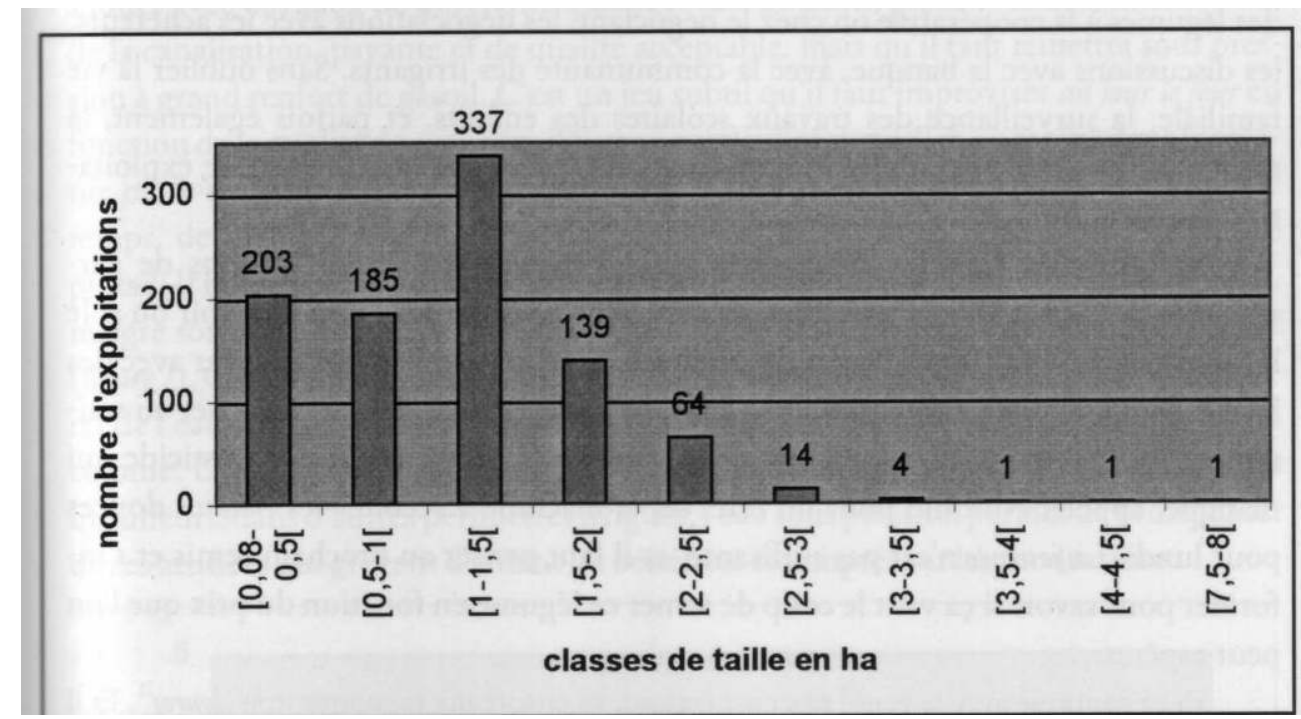

17 L'obsession de l'accès à la terre et à l'agrandissement de l'exploitation, presque naturelle de la part de petits agriculteurs dans une région marquée par l'injustice profonde du système latifondiaire a eu des effets pervers sur les rapports sociaux au quotidien, car dans ce lieu isolé, situé dans un «cul-de-sac » et où les gens semblent "enfermés ", les rancœurs et les frustrations héritées de cette histoire sont chaque jour rappelées par la rencontre et l'observation des voisins. De plus, cet agrandissement a créé une course en avant dans la charge de travail quotidien.

\section{Un quotidien harassant et plein d'aléas}

La vie quotidienne dans la colonie de Monte Algaida n'est pas de tout repos. Nous l'avons compris à l'évocation de cette histoire. Au présent, les agriculteurs insistent: il faut travailler tous les jours, de l'aube à la nuit, dimanche compris, en raison de la multitude de tâches qu'il faut assurer. Les vacances sont quasi inexistantes. Pour quel résultat?

\section{Répondre à une multitude de tâches pendant la journée}

La journée est atomisée entre de multiples tâches. Certaines concernent la production : les traitements, la récolte, la surveillance de l'eau et le réglage des systèmes d'irrigation, le déplacement des tuyaux, le calcul des mélanges entre l'eau des puits (pozo ou toyo) et de la réserve que chacun s'est faite avec l'eau de la canalisation, les contacts avec la coopérative pour le recrutement des manœuvres pour la récolte ou la commande de matériel ou d'un artisan qui laboure, etc. D'autres, la commercialisation : le transport des légumes à la coopérative ou chez le négociant, les négociations avec les acheteurs, les discussions avec la banque, avec la communauté des irrigants. Sans oublier la vie familiale, la surveillance des travaux scolaires des enfants, et parfois également, la recherche d'un travail complémentaire dans une bodega ou dans une grande exploitation qui va permettre de finir le mois.

Et tout cela dans la plus grande précipitation, avec des obligations de surveillance de l'irrigation et avec les yeux sur le ciel pour savoir s'il va pleuvoir ou si le levante va souffler. Alors il faudra protéger les carottes qui viennent de lever avec des bâches, 
qu'il faudra tenir verticales en bordure des planches de semis. Penser au traitement des œillets, aller au magasin de la coopérative pour acheter un pesticide qui manque, appeler Antonio pour lui réserver la machine à récolter les patates douces pour lundi. La journée n'est pas suffisante, et il faut penser au prochain semis et s'informer pour savoir si ça vaut le coup de semer ce légume en fonction du prix que l'on peut espérer...

Et ça continue avec la vente aux enchères qui va encore me rapporter trois douros ${ }^{11}$. Et il $\mathrm{y}$ a la réunion de la Comunidad de regantes où je vais m'énerver encore avec ce Rafael qui magouille toujours pour son intérêt, ... au fait j'ai oublié d'aller voir ce qu'ils ont fait avec le sable qu'ils doivent répandre sur la nouvelle parcelle dans les marais. Ils vont encore travailler comme des débutants parce que je ne serai pas là pour surveiller. [...] Tiens, voilà Pepe, qu'est-ce qu'il me veut celui-là, avec son tracteur qu'il a payé un monton ${ }^{12}$ d'euros; je parie qu'il va me demander de lui prêter mes bâches pour traiter son sol, [...] ou la pompe électrique que je viens d'acheter... ou bien il va me demander d'aller boire un coup chez Pablo et il va casser du sucre sur le dos de la coopérative qui s'en met plein les poches.

Ce discours imaginaire n'est pourtant pas très difficile à relier avec cette vie quotidienne des mille familles qui vivent désormais à La Algaida. Quand une tâche est terminée, il faut penser immédiatement à la suivante : le système des serres sous plastique impose de remplacer tous les deux ans les bâches, parce que la lumière intense dans le sud de l'Espagne et les intempéries rendent le plastique cassant. Aujourd'hui, il faut garder les bâches usées pour les confier à l'entreprise collective qui les traite, alors qu'auparavant on les mettait à brûler dans un coin de la colonie. En fait, on voit encore de ces tas de plastiques plus ou moins calcinés, ici et là. Chaque agriculteur a construit lui-même sa serre, avec des poteaux de bois tenus par des fils de fer et sur lesquels on tend les bâches. Travail exténuant : il faut avoir les bras continuellement en hauteur pour faire les épissures des fils de fer, sans avoir à monter sur un escabeau, car ça prend trop de temps et il faut aller vite.

\section{Organiser quotidiennement la distribution des eaux d'irrigation}

Cette vie quotidienne est rendue plus complexe à cause du système d'irrigation qui oblige à jouer avec des eaux de différente qualité : l'eau du puits, gratuite mais saline, et l'eau de la canalisation, payante et de qualité acceptable, mais qu'il faut remettre sous pression à grand renfort de gasoil. C'est un jeu subtil qu'il faut improviser au jour le jour en fonction de la qualité de ces eaux; il faut en particulier surveiller, au moins dans la colonie où le sol percole bien, les irrigations des voisins pour éviter d'irriguer en même temps, de manière à ce que les excédents d'eau du voisin réalimentent son propre puits $^{13}$. Il faut aussi prendre garde à ne pas trop attendre pour réinjecter l'eau du canal, malgré son coût, dans les réservoirs, faute de quoi la salinité de l'eau devient excessive (figure 7). Certains agriculteurs ont même installé des tuyaux qui permettent de transférer de l'eau de la colonie vers les marais et inversement des réservoirs des marais vers la colonie. Des milliers de kilomètres de tuyaux parcourent l'espace de la colonie, alors qu'ailleurs, dans d'autres périmètres irrigués, l'eau sous pression permet de se dispenser de ces artifices qui grèvent d'autant les bénéfices escomptés sur les productions. 
Figure 7. Fréquence d'utilisation de l'eau du canal et salinité des eaux d'irrigation à Algaida.

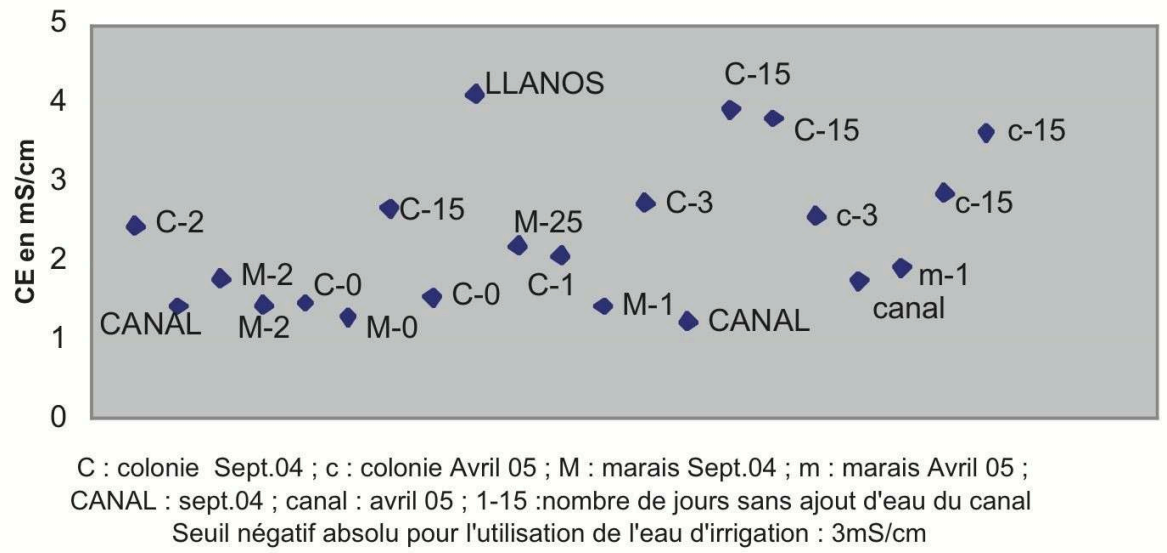

La gestion de l'eau est au centre des préoccupations des agriculteurs, mais elle complique la vie de tous les jours à cause de ce système hybride entre l'eau de la nappe et l'eau du bassin-versant. La surveillance de la qualité de l'eau n'est pas une chose aisée, car si la Comunidad de regantes effectue des mesures de la salinité de l'eau, elle ne le fait pas assez souvent ${ }^{14}$ et la salinité de l'eau est susceptible de varier assez rapidement. Souvent on doit faire confiance à la rumeur... et aussi se fier à ses propres capacités à évaluer l'humidité du sol, la salinité de l'eau (par observation des réactions des plantes, surtout) ainsi que les effets du vent qui sont toujours redoutés parce que ce dernier soulève le sable et érode en quelques minutes les jeunes plants tout juste levés.

Mais il reste une difficulté pour que se mette en place une conscience du risque hydraulique : la question de la pollution est le plus souvent évacuée ou carrément occultée, sauf à incriminer l'agriculture industrielle qui pollue l'eau du Guadalete. Les agriculteurs savent pourtant que les pesticides qu'ils utilisent dans les serres ou pour traiter les sols sont très toxiques, mais ils traitent assez souvent sans masque et évitent de parler des maladies de peau ou les affections ophtalmologiques ou pulmonaires évoquées par certains habitants non agriculteurs ${ }^{15}$ et apparemment tout aussi fréquentes dans la colonie. Il en va de même avec l'élimination des déchets qui reste désordonnée et peu organisée. De nombreux puits et réservoirs présentent aussi des signes d'eutrophisation, liés à l'utilisation des engrais azotés localement (figure 4) et en amont, dans le bassin du Guadalete ${ }^{16}$. Les effets à long terme de ces tâches du quotidien sont ainsi sous-estimés.

\section{Vendre les produits maraîchers : une saison de travail jouée en quelques minutes}

La commercialisation de la production est également une tâche complexe qui se trouve au bout de la chaîne de la multiplicité des tâches à effectuer. C'est celle qui conclue le cycle de production, qui détermine le revenu du travail et qui peut le remettre en cause, selon les prix qui sont pratiqués; car les prix varient quotidiennement eux aussi. Chaque mardi et jeudi ont lieu une vente aux enchères à la coopérative, à laquelle adhère la majorité des agriculteurs, les autres vendant leur production à des négociants privés ou à une société montée par quelques agriculteurs. L'autonomie complète de la 
vente est très rare, c'est le cas d'un agriculteur qui aurait passé un accord avec un détaillant, mais elle ne concerne jamais la production totale.

La subasta (vente aux enchères) se pratique à prix descendants : un agent commercial de la coopérative se place devant un lot de légumes et affiche un prix, le plus haut. Puis, à une vitesse que lui seul est en mesure d'assurer, il décline les prix en descendant, jusqu'à ce qu'un acheteur ${ }^{17}$ lui fasse un signe (imperceptible pour le non-initié). La vente est alors conclue. L'agriculteur peut alors faire le compte et savoir s'il a fait un bénéfice. Évidemment, tout le jeu des acheteurs consiste à laisser l'animateur descendre le plus bas possible pour acquérir le lot à un prix intéressant, au risque de voir le lot partir chez un concurrent. Pour les agriculteurs, cette vente aux enchères est une véritable épreuve; ils savent que c'est là que se joue en quelques minutes le revenu de leur travail de plusieurs mois. Et ce prix ne dépend pas que de la qualité du lot: il est fonction du prix du marché international que les agents commerciaux connaissent bien par le téléphone ou internet. Aussi les agriculteurs ne savent pas à quel prix leur production va être négociée et il arrive souvent que le prix retenu n'assure même pas le coût de revient de la production.

Le produit financier de la vente de la production n'est pas acquis à court terme par les agriculteurs : il faut attendre que la coopérative fasse le compte du débit (semences, pesticides et engrais, salaires des brigades de cueilleurs, notamment) pour le retrancher de la somme de la vente des légumes. Les factures ne sont pas soldées immédiatement par les acheteurs, notamment pour la production de carottes, qui passe par des intermédiaires. Il s'écoule donc un délai de quelques semaines à plusieurs mois entre la vente de la production et le paiement au producteur, période pendant laquelle il faut jongler au jour le jour avec les besoins du ménage et les autres en-cours.

\section{Jouer avec les institutions et les entreprises privées}

28 Comme on a pu le voir, les relations entre les agriculteurs et les institutions locales ne sont pas toujours claires. C'est là que des stratégies individuelles ou de petits groupes se déploient pour tenter de se faire une meilleure place dans le foncier, notamment avec la Comunidad de regantes. Par ailleurs, il faut savoir entretenir des relations cordiales avec les agents commerciaux ou les techniciens de la coopérative qui sont là pour apporter des conseils aux agriculteurs. Dans les entretiens avec les agriculteurs ou les agents de ces deux institutions, des informations paraissent parfois peu claires et il n'est pas aisé d'éclaircir totalement la réalité des enjeux. Il n'est pas difficile à l'inverse de deviner des jeux troubles entre les acteurs de cette colonie. Mais les entretiens ont leurs limites qu'il est quasi impossible de dépasser. Ils permettent en tout cas de bien mesurer la place qu'occupent les relations des agriculteurs avec les institutions dans la vie quotidienne et de percevoir leur crainte que de nouvelles contraintes viennent encore compliquer la vie de chaque jour. Parmi ces dernières, c'est désormais le changement climatique qui fait craindre une élévation des températures qui atteignent déjà un seuil impossible à surmonter: à la fin du mois de mai, certaines cultures deviennent impraticables sous serre en raison des températures qui peuvent atteindre 60 degrés. 


\section{Vivre en famille}

29 La plupart des agriculteurs vivent en famille dans la colonie. Pour l'agriculteur, il faut assurer de quoi faire vivre la famille et surtout le confort des enfants, leurs études, et chercher à imaginer un avenir pour eux. C'est le leitmotiv qui revient continuellement chez la plupart des agriculteurs: "Qu'est-ce que je vais bien pouvoir laisser à mes enfants?", disent-ils souvent. Peu d'espoir de voir la situation s'améliorer et le développement des cultures maraîchères au Maroc tout proche les inquiète, d'autant plus que ce sont souvent des grands propriétaires andalous qui s'installent de l'autre côté du Rif pour éviter les charges salariales trop élevées.

Pourtant, si la majorité d'entre eux vivent une situation difficile, les maisons qu'ils habitent sont de niveau de confort très hétéroclite. Du fait de la succession des générations, plusieurs maisons ont été construites dans les lots d'origine, empiétant sur les terres agricoles. Certains agriculteurs vivent encore dans les maisons d'origine, d'autres se sont fait construire des maisons fastueuses, entourées de murs. Dans le garage il n'est pas rare d'y apercevoir une voiture $4 \mathrm{x} 4$ de luxe. Comment expliquer ces disparités? Comment imaginer que ces agriculteurs parviennent à un tel niveau de revenu ? La rumeur donne une explication à cet état anachronique : certains habitants prétendent que ces agriculteurs riches vivent également du commerce de produits illicites que les pêcheurs rapportent au port de Bonanza proche. Mais il est évidemment difficile de vérifier. Cette situation occultée - mais vraisemblable - met encore une fois l'accent sur la difficulté de vivre au quotidien dans ce lieu où certains éléments du paysage rappellent à chacun les obstacles qui se dressent devant un espoir d'une meilleure condition sociale, en respectant une certaine morale.

\section{Conclusion : un quotidien aléatoire et peu prometteur}

31 La vie quotidienne de la Algaida paraît bien sombre. Le regard porté sur le passé n'est pas là pour enjoliver le présent : ce fut la misère et elle continue. Les récits des tâches qu'il fallait assurer « autrefois » sont toujours empreints de regrets et lourds de peines éprouvées, presque de honte, à l'inverse des récits enjolivés de la vie des campagnes heureuses d'antan. Le présent est vécu dans la crainte de l'aléa financier ou commercial, de l'accident de santé, d'une tempête qui pourrait tout dévaster. Ici, on pratique presque un métier d'équilibriste qui peut tomber à chaque instant, à chaque moment du jour présent. C'est bien le paradoxe de la situation des agriculteurs, très intégrés dans le marché, utilisant une technologie sophistiquée, alors que la plupart d'entre eux ne disposent que de très faibles surfaces. L'avenir ne porte que peu d'espoir. Peut-être les enfants s'en sortiront-ils? Mais eux-mêmes n'ont qu'à continuer : « No hay mas remedio " ${ }^{18}$.

Vivre au quotidien un milieu recomposé avec une telle intensité pourrait laisser croire qu'un tel artifice est, au contraire, mieux manipulable qu'un milieu « naturel ». Or, il semble bien que ce soit tout le contraire et que la vie quotidienne est soumise à des contraintes qui, au fur et à mesure qu'elles s'accumulent, restreignent sans cesse l'espace et le temps de liberté. Outre qu'il apporte la confirmation qu'un tel système comprime les temps naturels et les temps sociaux pour sacrifier à la loi du marché, cet exemple permet d'engager une réflexion sur la capacité des sociétés contemporaines à gérer des milieux que l'interventionnisme technique a radicalement transformés, au 
point que toute «culture collective du milieu ${ }^{19}$ » ne peut y trouver sa place. L'exercice au jour le jour de la gestion d'un tel milieu enseigne sur les limites de l'artificialisation du naturel pour des besoins dits « économiques ». Mais de quelle économie s'agit-il ? On peut aussi se questionner sur le rôle d'un tel système, fait de temps quotidien saturé et haché, de lente perte de sens de l'utopie égalitaire des débuts, sur les relations sociales au sein de cette colonie.

\section{BIBLIOGRAPHIE}

Ambrosy I. Garcia del Barrio, 1981, Curiosidades de San Lucar de Barrameda y de su comarca, t. 1, Ed. San Lucar.

Bejarano Palma R., 1997, Vegetación y paisaje en la costa atlántica de Andalucía, Univ. De Sevilla, Sec. de publicaciones.

Cohen M., Luginbuhl Y., 2005, « Small Irrigation and Sustainability of Water Resources (Andalusia - Spain) ", Proceedings of the 6th International Conference Ewra (European water resource association), 15 pages, ref. 086 (CD-Rom).

Grove A. T., Rackham O., 2000, The nature of Mediterranean Europe : an ecological history, New Haven Londres Yale Univ Press, 384 p.

Marquez C., 2005, Les paysages végétaux/agricoles du Bassin versant du fleuve Guadalete (Andalousie occidentale - Espagne). Une contribution à la question de la méditerranéité des paysages, Mémoire de master 2, Spécialité EMTS, MNHN-UMR Ladyss, 75 p. + annexes.

Ministerio de Medio Ambiente, 1994, Confederación Hidrográfica del Guadalquivir-Oficina de Planificación : 1994. Plan hidrológico del Guadalquivir, C.D. realizado por Kelvin Aplicaciones Multimedia.

Richards L. A., 1954, Diagnosis and improvement of saline and alkali soils, USDA Agric. Handbook $\mathrm{n}^{\circ}$ 60, Washington D.C., Dept. of Agric., 160 p.

Roldan Cruz C., 1994, Navaceros, nuevos agricultores y viñistas. Las estrategias cambiantes de la agricultura familiar, Sevilla, Fundación del Infante.

Torrejón y Boneta, D. Ángel de, 1914, Información sucinta relativa à la Colonia Agrícola del Monte Algaida de San Lucar de Barrameda, creada en virtud de la ley de colonización y repoblación interior de 30 de Agosto de 1907, mimeo $30 \mathrm{p}$.

Velásquez-Gaztelu J. P., 1994 [1760], Historia antigua y moderna de San Lucar de Barrameda, vol. II : Historia moderna de la Reconquista al reinado de Don Fernando VI (12641760), San Lucar de Barrameda, ASEHA.

\section{Données}

CHG, Area de Calidad de Agua, Ministerio del Medio Ambiente : Données sur la qualité des eaux, stations de Bornos, Hurones et Portal, années 2003 et 2004.

Communauté d'irrigants de Monte Algaida : Données sur la qualité des eaux (années 2004 et 2005), taille des propriétés (données anonymes). 
Bessard M., Larromanière M., Analyses de sols prélevés chez 15 agriculteurs d'Algaida en septembre 2004 et avril 2005 (Université Paris 7).

Cohen M., Bessard M., Marquez C., Mesures in situ de la qualité de l'eau chez 15 agriculteurs d'Algaida, septembre 2004 et avril 2005.

Cohen M., Marquez C., Campagne de géoréférencement et de mesure de la qualité des eaux le long du cours et du canal du Guadalete, avril 2005.

\section{NOTES}

1. Programme «Crique » sur la perception du risque hydraulique en Méditerranée, UMR Ladyss, comparaison de plusieurs territoires irrigués en France, Espagne, Tunisie, Maroc, Portugal (Cohen et Luginbühl 2005).

2. Cette petite ville est connue des amateurs de vin pour être le lieu de production de la Manzanilla, vin cher à Carmen de Bizet qui en dégustait sous les remparts de Séville.

3. Le système de la dehesa consiste à aménager un terrain boisé de façon à y mener simultanément une activité pastorale et une exploitation du bois (souvent du charbonnage). La physionomie de ces terrains s'apparente à celle d'une savane agro-forestière, constituée d'une strate herbacée et d'arbres dispersés (Grove et Rackham, 2000).

4. Technique utilisée dans les jardins maures. On peut s'interroger sur l'origine de cette technique qui fut vraisemblablement répandue en Andalousie pendant la période arabe qui prit fin ici au XIII ${ }^{\mathrm{e}}$ siècle. Le mot même de Algaida est d'origine arabe, et signifie selon les sources terrain boisé ou grand monticule de sable (Ambrosy, 1981).

5. En témoigne une granulométrie très homogène entre 10 et $80 \mathrm{~cm}$ de profondeur (les sables assez fins, entre 200 et $500 \mu$, représentent 70 à $80 \%$ des particules selon les lieux échantillonnés).

6. Les carottes, plantées à l'automne, sont la principale culture d'exportation qui ne peut être conduite sous serre.

7. Des produits très toxiques, interdits par l'Union européenne, continuent à être utilisés par les agriculteurs de la Algaida, qui ont obtenu une dérogation.

8. La famille de Médina Sidonia est l'une des plus grandes familles latifondiaires de l'Andalousie. Elle est aussi connue par la «Duchesse rouge » (Duquesa de Medina Sidonia) qui se distingua par son appartenance au parti communiste espagnol et par sa position en faveur de la défense des ouvriers agricoles du vignoble. Elle fut emprisonnée sous Franco. Elle vit encore à San Lucar de Barrameda dans un palais où une bibliothèque rassemble de nombreux documents notariés sur la dynamique du foncier en Andalousie, notamment.

9. Certaines familles auraient été déplacées du nord de l'Espagne : il s'agissait de syndicalistes qui s'étaient distingués dans des grèves ouvrières ou par leur action militante particulièrement intense. Leur déplacement était un moyen de mettre fin à la cohésion syndicale.

10. Ces «sablières » ont donné lieu à la création d'étangs en raison de la proximité de la nappe phréatique ; ces étangs sont aujourd'hui des réserves « naturelles » protégées (figure 4) et entrant dans la zone périphérique du Parc National de Doñana, le plus grand parc national d'Espagne.

11. Un douro correspond à trois pesetas; les agriculteurs comptent encore souvent en pesetas et non en euros.

12. Un monton: une somme énorme.

13. Lors de l'irrigation sur une parcelle voisine, on peut observer parfaitement l'eau qui suinte le long des parois du puits et qui fait remonter son niveau. 
14. Ces mesures ont été réalisées assez régulièrement par une technicienne de la Comunidad de Regantes; depuis son départ, les mesures proviendraient des services techniques extérieurs (figure 3).

15. Selon en particulier des membres de l'association des habitants de la Algaida, il y aurait de nombreux cas de ces affections chez les agriculteurs.

16. D'après des mesures mensuelles en 2003 et 2004, la teneur en nitrate des eaux dépasse au Portal $20 \mathrm{mg} / \mathrm{l}$ un mois sur deux, $50 \mathrm{mg} / \mathrm{l}$ un mois sur 10, alors qu'elle est inférieure à $10 \mathrm{mg} / \mathrm{l}$ en amont de Jerez dans le bassin versant du Guadalete.

17. Ces acheteurs sont souvent des intermédiaires de négociants qui font le commerce des légumes sur de grands volumes et les revendent aux chaînes de supermarchés en Europe du Nord.

18. «Il n'y a pas d'autre moyen » (que de continuer à trimer).

19. On entendra ici par «culture collective du milieu » la connaissance empirique que la société locale se forge par observation des processus biophysiques.

\section{RÉSUMÉS}

L'exemple de la Colonie agraire de Monte Algaida (Andalousie occidentale) est l'occasion d'une relecture de l'histoire d'une communauté rurale, de la transformation radicale qu'elle a opérée sur son milieu naturel, mais aussi de l'évolution de ses relations sociales, à travers le prisme du quotidien. L'histoire de la transformation des terres apparaît ainsi comme le résultat d'une accumulation de tâches quotidienne, d'un labeur obstiné, même s'il a changé de nature avec la mécanisation et l'aménagement de nouvelles terres. Cette communauté, assez isolée géographiquement, s'est développée numériquement depuis son installation, dans un cadre très contraint par la Loi de colonisation agraire, mais elle s'est aussi fragmentée, divisée, entre ceux qui ont su (ou pu) profiter de la modernisation et des politiques d'aménagement, et ceux qui sont restés en marge, engendrant une coexistence difficile au quotidien. Ces difficultés sont d'autant plus ressenties qu'elles font écho, ou pourrait-on dire système, avec une vie morcelée en de multiples tâches, destinées à la production, la commercialisation, à la vie de famille, entre lesquelles on ne peut arbitrer. C'est bien le paradoxe de la situation de ces agriculteurs, très intégrés dans l'économie de marché, s'appuyant sur un système technique sophistiqué, et pourtant disposant de surfaces minuscules. L'exercice au jour le jour de la gestion d'un tel milieu enseigne sur les limites de l'artificialisation du naturel pour des besoins dits " économiques ».

The example of the Monte Algaida farmers' colony (Western Andalucia) offers the opportunity to review the history of a rural community, the radical transformation that it has wrought in its natural environment, and also the evolution of its social relations through the prism of everyday life. The history of land transformation appears to result from an accumulation of daily tasks, obstinate labour, even though its nature has changed since mechanization has started, and new land has developed. This community, which is rather isolated from a geographical point of view, has grown since it first was established under a constraining law on agricultural colonization, but it also became fragmented and divided between those who knew how (or were able) to benefit from modernization and development policies, and those who stayed on the side, which led to a difficult daily co-existence. These difficulties are all the more felt as they echo, or shall we say interact, with a life split among several tasks aimed at production, commercialisation, family life 
among which it is difficult to arbitrate. This is where the situation of these farmers becomes paradoxical. For if they are very much integrated in the market economy thanks to a sophisticated technical system, they still have tiny areas at their disposal. The exercise of managing such an environment on a daily basis shows the limits of rendering nature artificial for the sake of so-called "economic" needs.

INDEX

Index géographique : Espagne

Mots-clés : quotidien, espace rural, agriculture, économie libérale, aménagement rural

\section{AUTEURS}

\section{YVES LUGINBÜHL}

Directeur de recherche,

Ladyss,

CNRS/univ. de Paris 1,

2 rue Valette,

75005 - Paris

luginbuh@univ-paris1.fr

\section{MARIANNE COHEN}

Maître de conférences en géographie,

Ladyss,

CNRS/univ. de Paris 7

cohen@univ-paris-diderot.fr 